\title{
Study on the Development of a New Device with Dual Cameras for Evaluating Expiratory Nasal Flow
}

\author{
Rieko Doi,* Shuko Akagami, † Katsuya Kondo, \\ Tomoki Kataoka,* Takashi Narai,* Nobuyuki Fujii,* Makoto Kawasaki,* Kazuma Otsuki* and Isamu Kodani* \\ *Division of Oral and Maxillofacial Surgery, Department of Sensory and Motor Organs, School of Medicine, Faculty of Medicine, \\ Tottori University, Yonago 683-8504, Japan, †Division of Oral and Maxillofacial Surgery, Tottori University Hospital, Yonago 683- \\ 8504, Japan, †Department of Electrical Engineering and Computer Science, Tottori University, Tottori 680-8550, Japan, \$Department \\ of Oral and Maxillofacial Surgery, Matsue Red Cross Hospital, Matsue 690-8506, Japan, and IDepartment of Oral and Maxillofacial \\ Surgery technology, Division of Medical Technology, Tottori University Hospital, Yonago 683-8504, Japan
}

\section{ABSTRACT}

Background Use of the Glatzel mirror for measuring expiratory nasal flow in preschool children has the disadvantage of vagueness, and the mirror may induce fear and inhibition of interest in those children. In response to these limitations, we developed a new device with dual cameras for measuring expiratory nasal flow in 2 to 6 year old children. The aim of this study is to compare the Glatzel mirror and the new device, in terms of accurate assessment of expiratory nasal flow, children's feelings, and correlation to each child's profile.

Methods This study evaluated 20 cleft lip and palate patients and 21 healthy children aged between 2 and 6 (under 7) years. After consent was granted, a 4-week screening period was undertaken followed by inspection at weeks $8,16,24$, and 32. Each inspection was conducted while the children were asked to pronounce various sounds and comprised three stages: i) use of the Glatzel mirror, ii) subjective visual assessment using the new device, and iii) image recording by dual cameras of the new device. Questionnaires for the new device were administered at the initial and final inspections. To contrast the results between the Glatzel mirror and the new device, the numbers that indicated values of subjective visual assessment and camera assessment greater than the assessment values of the Glatzel mirror were compared. For measuring the children's responses to the new device compared with those to the Glatzel mirror, the answers to the questionnaires were compared. For the comparison of the children's profiles (age and sex) and feelings, the numbers of subjects who could use the new device were measured.

Results The camera assessment of the new device indicated significantly greater values than that of the Glatzel mirror $(P<0.05)$. The feelings of the subjects to the new device mostly improved as the study progressed. Subjects aged 3 years and older were generally able to use the new device from the initial inspection. For both sexes, as the inspection progressed, the number occasions of successful use increased.
Conclusion This study demonstrated the superiority of the new device with dual cameras to the Glatzel mirror in terms of functionality and attitude of children.

Key words expiratory nasal flow; Glatzel mirror; speech disorders; speech therapy; velopharyngeal insufficiency

Velopharyngeal insufficiency (VPI) after palatoplasty in patients with cleft lip and palate is a significant problem that children face from an early stage after the procedure. ${ }^{1-4}$ The treatment of VPI is dependent on the severity of the disease, ${ }^{1,5,6}$ and accurate diagnosis is extremely important for the child's quality of life and their future language and speech abilities..$^{7-9}$

Various techniques, all of which have their advantages and disadvantages, are used to evaluate VPI and determine the most appropriate treatment..$^{10}$ Postoperative therapy is undertaken in both clinical and non-clinical environments. It can be conducted in various medical, educational, healthcare, and welfare facilities and the evaluation methods may vary between facilities. ${ }^{7}$ Given the numerous variables related to effective postoperative therapy and the environment of the child, the benefits of using the Glatzel mirror (GM) to measure expiratory nasal flow include practicality and convenience of use, adaptability to the environment (i.e., it is portable and functional in clinical and non-clinical environments), non-invasiveness, and ability to be used by both medical professionals and laypeople. However, in clinical conditions, the GM used in the assessment of expiratory nasal

Corresponding author: Rieko Doi, DDS, PhD

rieko3@tottori-u.ac.jp

Received 2020 June 3

Accepted 2020 August 31

Online published 2020 September 28

Abbreviations: CA, camera assessment; FAS, full analysis set; GM, glatzel mirror; ND, new device with dual cameras for evaluating expiratory nasal flow; Q., question; SVA, subjective visual assessment; VI, visual inspection; VPI, velopharyngeal insufficiency 
flow may induce fear in children due to the mirror's reflection and induce inhibition of their interest due to its passive design. The limitations of the GM test are that the results obtained are vague because the mirror does not regulate the flow of condensation and the short time frames for evaluation lead to measurement errors. In response to the limitations of the GM test, we developed a device consisting of two digital cameras with tulip ornamentation to evaluate expiratory nasal flow.

The objective of this study was to accurately assess expiratory nasal flow by a new device with dual cameras with tulip ornamentation, and to evaluate the children's attitude towards the new device by the following methods: i) comparing the assessment results between the GM and the new device with two digital cameras for evaluating expiratory nasal flow (ND), ii) measuring the attitude of the children towards the ND compared to that towards the GM, iii) measuring the correlation between the children's profile (age and sex) and attitude towards the ND.

The study was approved by the clinical study ethic screening committee (No. CRB618005) of Tottori University Hospital (No. C17B007) and was performed with the approval of the Japanese Ministry of Health and Welfare (jRCTs062180020).

\section{SUBJECTS AND METHODS Subjects}

This study was a prospective, single facility, interventional, non-randomized, non-blinded, parallel-group comparison study. The study was undertaken in the period between September 29, 2017 and January 22, 2020. All cleft lip and palate patients underwent medical treatment, including palatoplasty, at the Division of Oral and Maxillofacial Surgery, Department of Sensory and Motor Organs, School of Medicine, Faculty of Medicine, Tottori University. The procedure for all patients was the Wardill pushback method.

\section{Inclusion/ exclusion criteria}

This study had two groups: a cleft lip and palate group and a healthy children's group. The inclusion criteria for the cleft lip and palate patients were as follows: i) both male and female patients, ii) children aged 2 to 6 years (under 7 years), iii) patients who underwent palatoplasty, and iv) patients who had no other underlying disease that would affect the outcome of the research. The inclusion criteria for healthy children were as follows: i) both male and female children, ii) aged 2 to 6 years (under 7 years), iii) children who did not have cleft lip and palate, iv) children who had no other underlying disease that would affect the outcome of the research.
The exclusion criteria for the research included the following: i) children for whom consent was not acquired, and ii) children considered by the research team to be ineligible due to learning difficulties and developmental difficulties or with other preexisting conditions.

\section{Case diagram}

Initially, consent was acquired from 24 cleft lip and palate patients, and 55 healthy children. The registered cases represented 21 cleft lip and palate patients and 46 healthy children. Among these, three cleft lip and palate patients were disqualified, two cases did not conform to the inspection and 1 case moved out from their place of residence. On the other hand, nine healthy children were disqualified as they did not conform to the inspection. From the registered 67 cases, 26 cases did not meet the inclusion criteria; 1 cleft lip and palate patient and 16 healthy children were disqualified for not meeting the inclusion criteria, and 9 healthy children graduated from nursery school. In this way, the Full Analysis Set (FAS), i.e., the total cases for analysis contained 41 cases. Namely, the FAS consisted of 20 cleft lip and palate patients and 21 healthy children (Fig. 1).

There were 6 males and 14 females in the cleft lip and palate patient group. According to the age distinction, four children were aged 2 years, four were aged 3 years, three were aged 4 years, four were aged 5 years, and five were aged 6 years. The average age of the subjects was 4.1 years. The standard deviation of age was 1.48 years. Of the 21 healthy children, there were 11 males and 10 females. Of these, nine children were aged 2 years, four were aged 3 years, four were aged 4 years, four were aged 5 years, and there were no children of 6 years of age. The average age of the children was 3.1 years. The standard deviation of age was 1.17 years.

The subjects were classified into four cleft types, including three cases of bilateral cleft lip and palate, six cases of unilateral cleft lip and palate, six cases of cleft of the hard and soft palate, and five cases of cleft of the soft palate. One case was previously diagnosed with Pierre Robin syndrome and ventricular septal defect. Four cleft lip and palate patients simultaneously suffered with alternative disorders, including two cases of hypospadias, one case of exotropia, and one case of hemangioma.

\section{Study design}

Appropriate consent was obtained from the subjects' guardians followed by a 4-week screening period, and a 32-week observation period (Fig. 2). The inclusion and exclusion criteria were established and the subjects were 


\section{【Cleft lip and palate patients】}

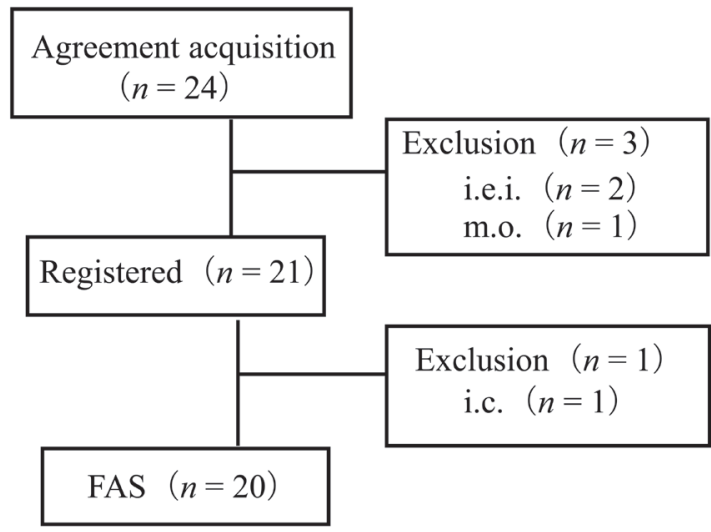

【Healthy children】

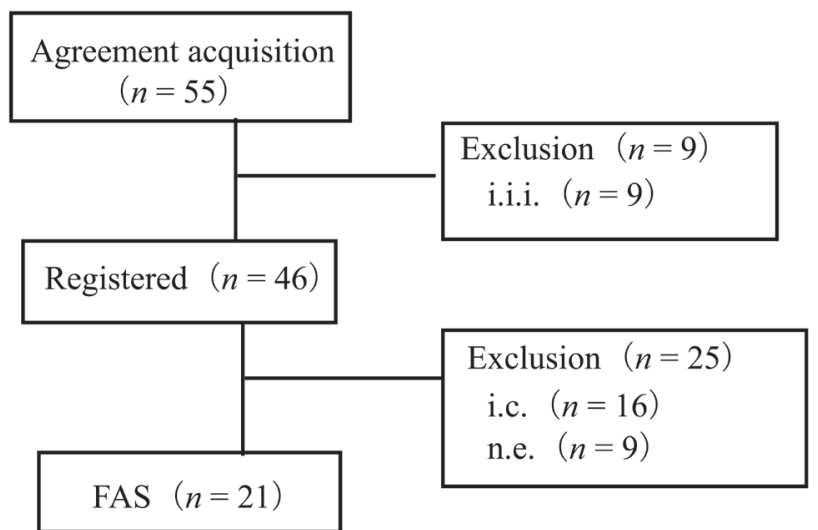

Fig. 1. Case diagram. The FAS, i.e., the total cases for analysis, consisted of $20 \mathrm{cleft}$ lip and palate patients and 21 healthy children. FAS, full analysis set; i.c., incompatible condition; i.e.i., irregular examination interval; i.i.i., irregular inspection interval; m.o., moved out; n, number of cases; n.e., nursery school exit.

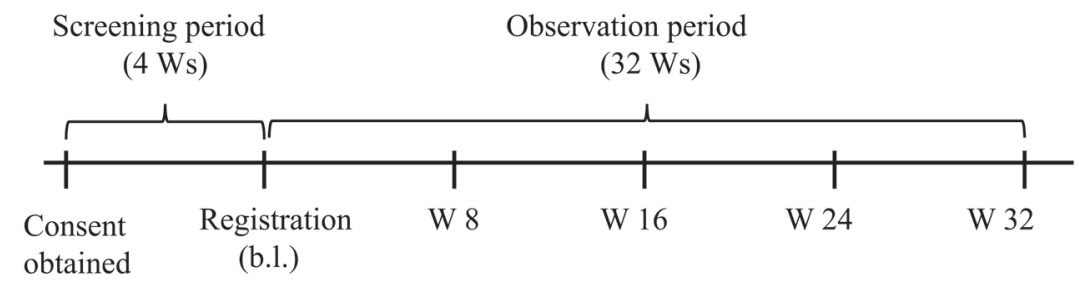

Fig. 2. Study design. The study consisted of a screening period of $4 \mathrm{Ws}$ and an observation period of 32 Ws. b.l., base line; W, week.

confirmed to comply with the criteria. The subjects were then registered and inspected, and evaluated at weeks $8,16,24$, and 32 . The questionnaire concerning patients' feelings towards the use of the ND were administered at the initial and final inspection (Fig. 3). A tolerance range of \pm 7 weeks on each inspection date was permitted. The maximum participation period of the study was 36 weeks. Each assessment took approximately 15 minutes.

\section{Equipment}

We created the ND for the purpose of use in children. The ND concealed two digital cameras in a colored tulip ornamentation, and the mirror was enclosed in a plastic casing in order to reduce the sense of fear among children. ${ }^{11}$ The outside of the ND was coated with a non-toxic paint, (NTX ultruck kisyou flat, Osaka Toryo Kogyo Co., Ltd., Osaka, Japan), a paint used for food trays (Fig. 4).

During inspection of VPI, expiratory nasal flow from the nostrils appears as condensation on the GM. The 2 digital cameras, embedded on either side of the yellow tulips, were used to record the condensation.
The assessment image was obtained by these two different viewpoints. The shutter speed of the camera was 10 frames per second and weight was about $350 \mathrm{~g}$, including battery. The orange tulips were equipped to stimulate the children's interest. Figure 5 shows original images by the ND. The upper left photo shows image from the left camera and the upper right photo shows image from the right camera. The lower middle photo shows the image after the processing. A breath image was acquired from the right and left original distorted images by using the self-similarity principle. ${ }^{12,13}$ The red arrow in Fig. 5 shows the maximum value of expiratory nasal flow as condensation on the mirror (arrow). Then the results were analyzed for this study.

\section{Inspection parameters and assessment methods}

The evaluation of cleft lip and palate patients was performed at the speech training room of Tottori University Hospital. The evaluation of healthy children control group was conducted at the hospital nursery school, Suginoko. All inspections were undertaken by a single speech therapist to ensure consistency in analysis. For all inspections, the GM and ND were used and the 
Q. 1. How do you feel about the ND compared to the GM?

1) E; Excellent, 2) G; Good, 3) P; Possible, 4) I; Impossible, 5) B; Bad

Q. 2. Did you feel scared about the ND?

1) I did not feel scared, 2) I did not feel so scared, 3) Neither,

4) I felt little scared, 5) I felt scared

Q. 3. Did you use the ND well?

1) Very well, 2) Little well, 3) Neither, 4) Little bad, 5) Very bad

Q. 4. Do you want to use the ND again?

1) I want to use it again, 2) I want to use it, 3) Neither,

4) I do not want to use it, 5) I do not want to use it again

Fig. 3. Questionnaire about the ND. The questionnaire about the ND was administered at the initial and final assessment. GM, glatzel mirror; ND, new device with dual cameras for evaluating expiratory nasal flow. Q., question.
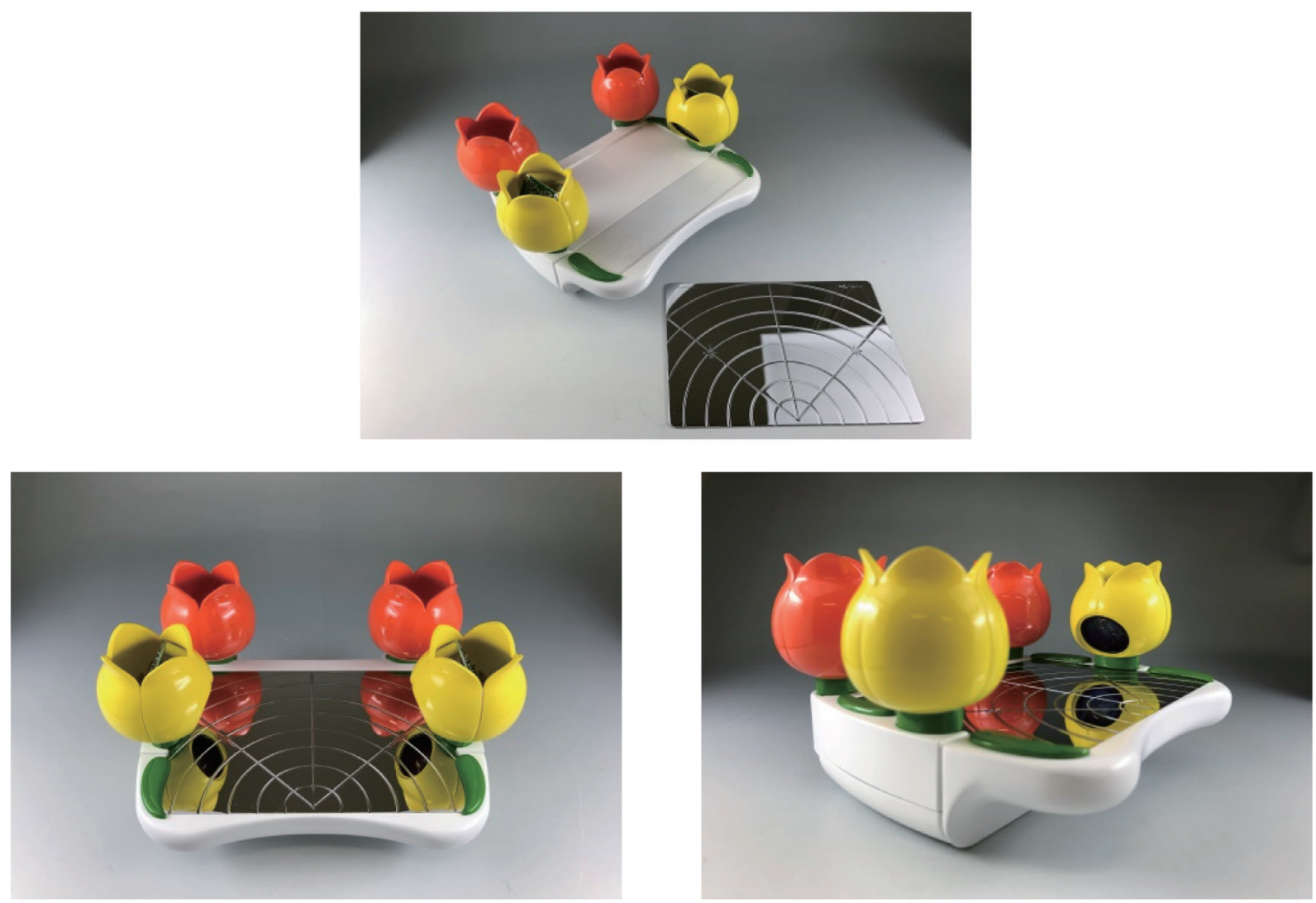

Fig. 4 . New device with dual cameras for evaluating expiratory nasal flow. The two digital cameras were embedded on either side of the yellow tulips and used to record the condensation. The orange tulips were equipped to induce children's interest. 

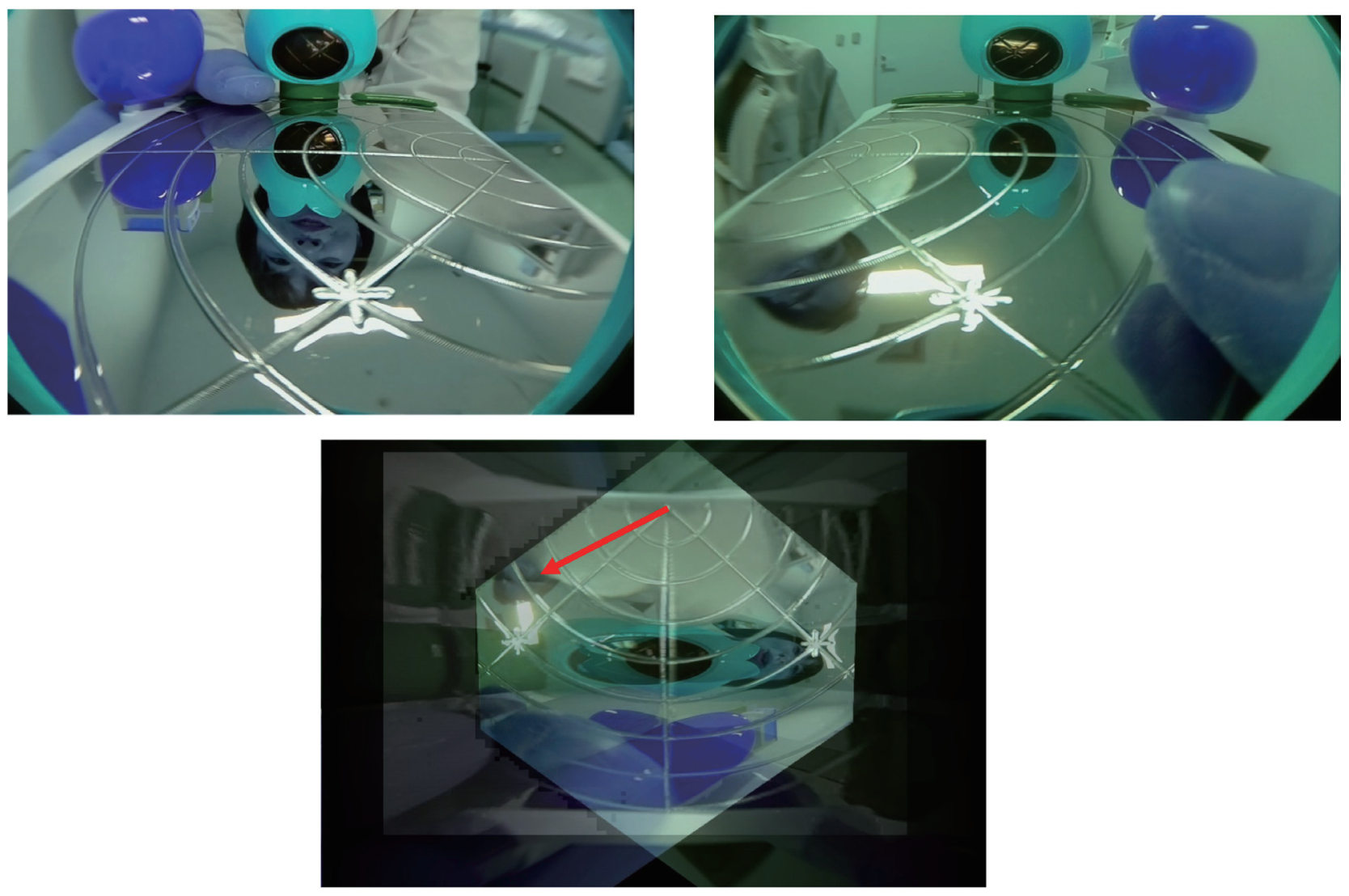

Fig. 5. Original images recorded by the new device with dual cameras. The red arrow of the lower middle photo shows the maximum value of expiratory nasal flow as condensation on the mirror (arrow).

analysis was conducted by placing the apparatus under the patient's nostrils. The subjects performed the following tasks during inspection: i) sound pronunciation, ii) straw test for soft blowing, or party horn test for hard blowing. At the beginning of each assessment, GM assessment was undertaken; thereafter, subjective visual assessment (SVA) and camera assessment (CA) were undertaken using the ND. In case of healthy children, if they could pronounce vowel sound exactly, they had the permission for omitting subsequent inspection in consideration of the subjects' concentration and inspection time.

Sound pronunciation: Participants were asked to articulate the following sounds: i) vowel sounds (a, i, u, $\mathrm{e}, \mathrm{o})$, ii) consonant sounds (pa, shi), iii) nasal sound (nu), and iv) sentence (aoi ie wa iiyo). If the timing of sound pronunciation and expiatory nasal flow was not correct, the inspection was repeated several times. In the softblowing test, total $5 \mathrm{~cm}(110 \mathrm{~mL})$ of water was placed in a paper cup, and the GM or the ND was placed under the subject's nose and the subject was instructed to inhale and then gently exhale through the straw for as long as possible. The hard-blowing test was undertaken only when subjects had difficulty in soft blowing. It included using a party horn hardly for as long as possible.

In of many these tests, we scaled the longest foggy length on the mirror of the GM, SVA of the ND, and CA of the ND, and these were treated as the assessment values respectively. For example, the assessment value of the case in Fig. 5 was the length of the red arrow (3.5). We measured the assessment values of the same test words by using three methods, GM, SVA of the ND, and CA of the ND.

To investigate the superiority of the ND against the GM, we compared the assessment value of SVA of the ND or CA of the ND with that of the GM. In case the assessment value of SVA of the ND or CA of the ND was longer than the assessment value of GM, we considered it as valuable for this study. In contrast, if the assessment value of SVA of the ND or CA of the ND was not longer than the GM, it was considered inadmissible. For example, when the subject pronounced the same vowel sound "a", the assessment value of GM was 1 , the SVA of the ND was also 1 (except GM $<$ SVA), and the CA of the ND was also 1 (except GM $<$ CA), this case indicated that the ND detected the same 
assessment value as the GM and it was not superior to GM. Contrarily, if the assessment value of GM was 1, the SVA of the ND was also 1 (except GM $<$ SVA), but the CA of the ND was $2(\mathrm{GM}<\mathrm{CA})$, this case indicated that the CA of the ND detected greater than the GM and it was considered that the CA of the ND was superior to GM. In another case, for example, if the assessment value of the GM was 1 , the SVA of the ND was 2 (GM $<$ SVA), and the CA of the ND was also 2 (GM $<$ $\mathrm{CA})$, this indicated that not only the CA but also SVA detected greater assessment values than the GM. In this case, we did not consider that the CA of the ND was clearly superior to the GM. In another case, the assessment value of GM was 1 , the SVA of ND was $2(\mathrm{GM}<$ $\mathrm{SVA}$ ), and the CA of ND was 1 (except $\mathrm{GM}<\mathrm{CA}$ ), this indicated that the CA of the ND was not greater than the assessment value of the GM, and it was not superior to GM. Thereafter, we counted the number of cases in these four patterns, and compared the assessment values of the SVA and CA of the ND with that of the GM using statistical analysis.

Variance in the assessment values was evaluated by comparing the values of the SVA and CA of the ND to that of the GM.

At the initial and final inspection time, a questionnaire was administered. The details of contents are indicated in Fig. 3. The questionnaire was administered in interview format and the answers were documented. Regarding each questionnaire contents, the answers were calculated as percentage of the number of cases and compared. In the healthy children control group, subjects that successfully completed assessments and responded satisfactorily to the questionnaire did not have to redo the assessment.

For measuring the correlation between the subjects' profile (age and sex) and attitude towards the ND, the number of subjects that could use the ND were calculated and indicated as percentage in each case.

Any injuries or illness resulting from this research were reported as an adverse event.

\section{Statistical Analysis}

Statistical analysis was performed using Excel 2016 (Microsoft, Redmond, WA), and EZR (R for Mac OS X cocoa). The correlation of assessment results between the GM and the ND was calculated using McNemar test. Paired test was used for comparisons between pairs of inspection variables. $P$-values of $<0.05$ were considered to be statistically significant.

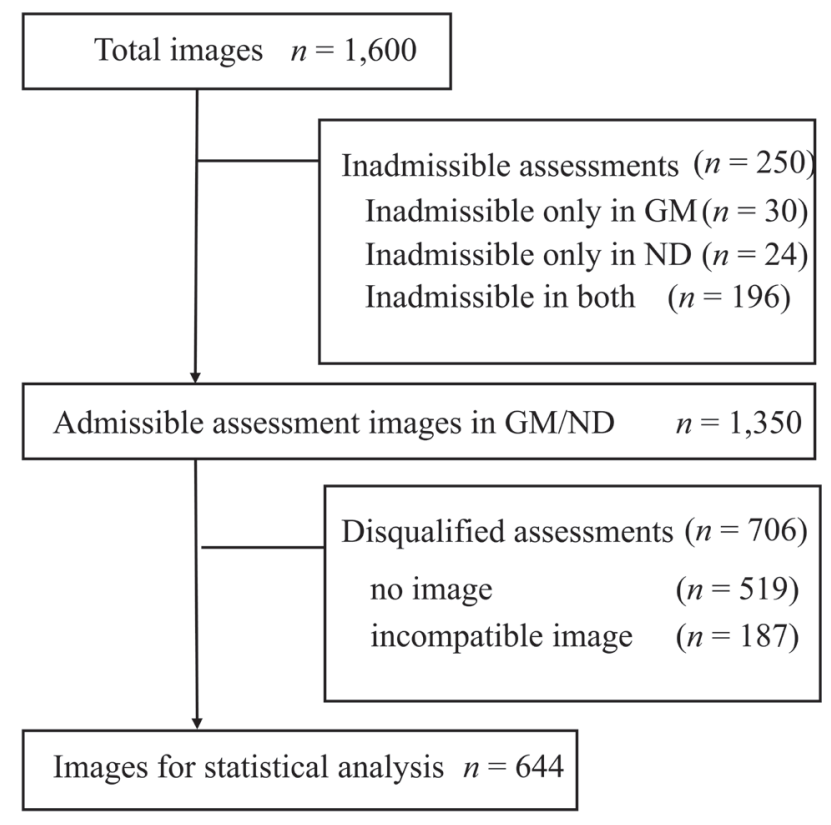

Fig. 6. Number of the images used for statistical analysis. GM, Glatzel mirror; ND, new device with dual cameras for evaluating expiratory nasal flow; $n$, number of cases.

\section{RESULTS}

The difference in general conditions between cleft lip and palate patients, and healthy patients were not acknowledged in this study.

\section{Contrasting the assessment results between the GM and the ND}

The research was based on total 1,600 images. Of the 1,600 images assessed, 250 image assessments were inadmissible (30 image assessments were inadmissible only in the GM, 24 images were inadmissible only in ND, and 196 images were inadmissible in both). Children's inability to comprehend assessment instructions and their mood were attributed to assessment failures. A total number of 1,350 GM and ND assessment images were admissible for analysis. Among those, 519 images had no expiratory nasal leakage. About 187 images did not meet measurement requirements, 186 images were blurred, and 1 image was misaligned. As a result, further 706 images were excluded. Finally, 644 images were considered admissible for analysis in this study (Fig. 6).

The results of comparison of assessment values between the GM and the SVA or CA of the ND was summarized (Fig. 7). In detail, the assessment values of SVA and CA of the ND were not longer than those of the GM in 363 cases. These cases indicated that the ND was not superior to the GM. In 73 cases, the assessment 


\begin{tabular}{|c|c|c|c|}
\hline \multicolumn{2}{|c|}{} & \multicolumn{2}{|c|}{ CA } \\
\cline { 3 - 4 } \multicolumn{2}{|c|}{} & $\begin{array}{l}\text { except GM }<\text { CA } \\
(\mathrm{GM}>\text { CA or GM }=\text { CA })\end{array}$ & $\mathrm{GM}<\mathrm{CA}$ \\
\hline \multirow{2}{*}{ SVA } & $\begin{array}{l}\text { except GM }<\text { SVA } \\
\text { (GM }>\text { SVA or GM }=\text { SVA })\end{array}$ & 363 & 73 \\
\cline { 2 - 4 } & GM $<$ SVA & 39 & 169 \\
\hline
\end{tabular}

$(n)$

Fig. 7. Comparison of assessment values between GM and SVA of the ND or CA of the ND. In 73 cases, the results indicated that the CA of the ND was superior to the GM. CA, camera assessment; GM, Glatzel mirror; n, number of cases; ND, new device with dual cameras for evaluating expiratory nasal flow; SVA, subjective visual assessment.

values of the CA of the ND were longer than those of the GM, but the assessment values of the SVA of the ND were not longer than the GM values. These cases indicated that the CA of the ND was superior to GM. In 169 cases, the assessment values of the SVA and CA of the ND were both longer than those of GM. In these cases, it was considered that the CA of the ND was not clearly superior to GM. Remaining 39 cases showed that the assessment values of the SVA were longer than the GM, but the CA values of the ND were not longer than those of the GM. These cases indicated that the CA of the ND was not superior to the GM. We analyzed these results by using the McNemar test, which indicated that the CA was significantly more accurate at assessing expiratory nasal flow images than the GM. (McNemar's $\chi^{2}=9.7232, \mathrm{df}=1, P=0.00182$ ).

Regarding the variance of assessment values between the SVA and CA of the ND and the GM, no significant difference was observed (SVA; median 0, average 0.054 , CA; median 0, average 0.053) (Fig. 8).

\section{Measuring the attitude of subjects towards the ND compared to the GM}

We conducted the questionnaire about the ND and compared it with the GM at the initial and final inspection. It indicated that the feeling of subjects towards the ND. All graphs show the applicable numbers in \% (Figs. 9-13).

As the assessment progressed, cleft lip and palate patients responded increasingly positively to "Question (Q.) 3: I used very well" and "Q. 4: I want to use it again". The subjects' positive feelings to use the ND [as Q. 1: excellent / good / possible] decreased slightly between the initial and final inspection, but with no

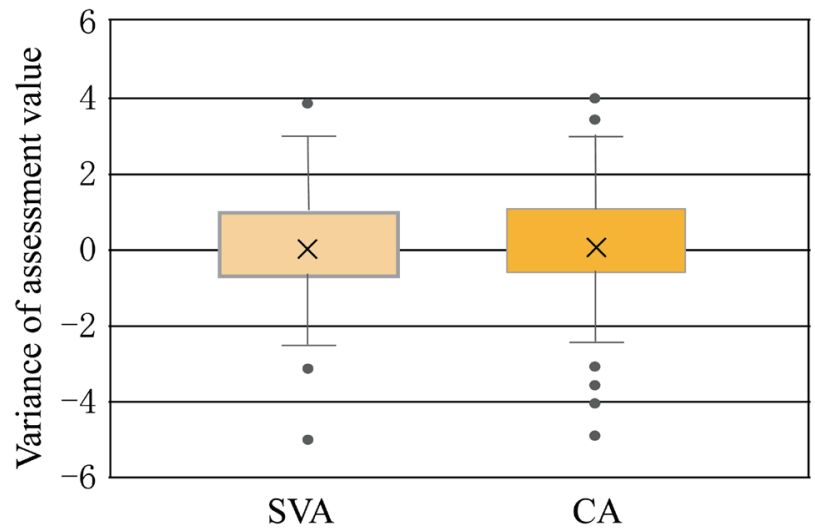

Fig. 8. Variance of assessment values between SVA and CA of the ND and GM. There was no significant difference between the SVA and CA of the ND and the GM. CA, camera assessment; GM, Glatzel mirror; ND, new device with dual cameras for evaluating expiratory nasal flow; SVA, subjective visual assessment.

significant difference. No cleft lip and palate patient responded to "Q. 2" as "I felt scared" after the final assessment (Fig. 9).

As testing progressed, healthy children's positive feelings to use the ND [as Q. 1; excellent / good / possible] increased. And healthy children also responded increasingly positively to "Q. 3" as" I used it very well", and to "Q. 4" as "I want to use it again". No healthy child responded to "Q. 2" as "I felt scared" during the assessment (Fig. 10).

\section{Measuring the correlation between subjects' pro- file (age and sex) and attitude to the ND}

We examined how the ND was received according to age- or sex-specific attitude by the subjects. We aimed 


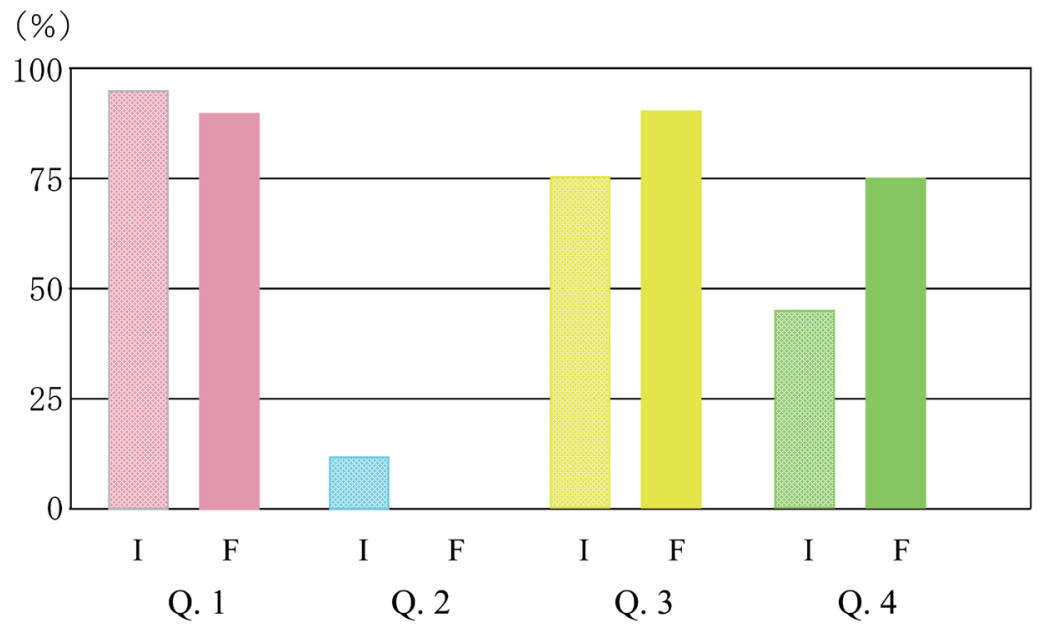

Fig. 9. Cleft lip and palate patients' attitude towards the ND compared to GM. F, final inspection; GM, Glatzel mirror; I, initial inspection; ND, new device with dual cameras for evaluating expiratory nasal flow; Q., question.

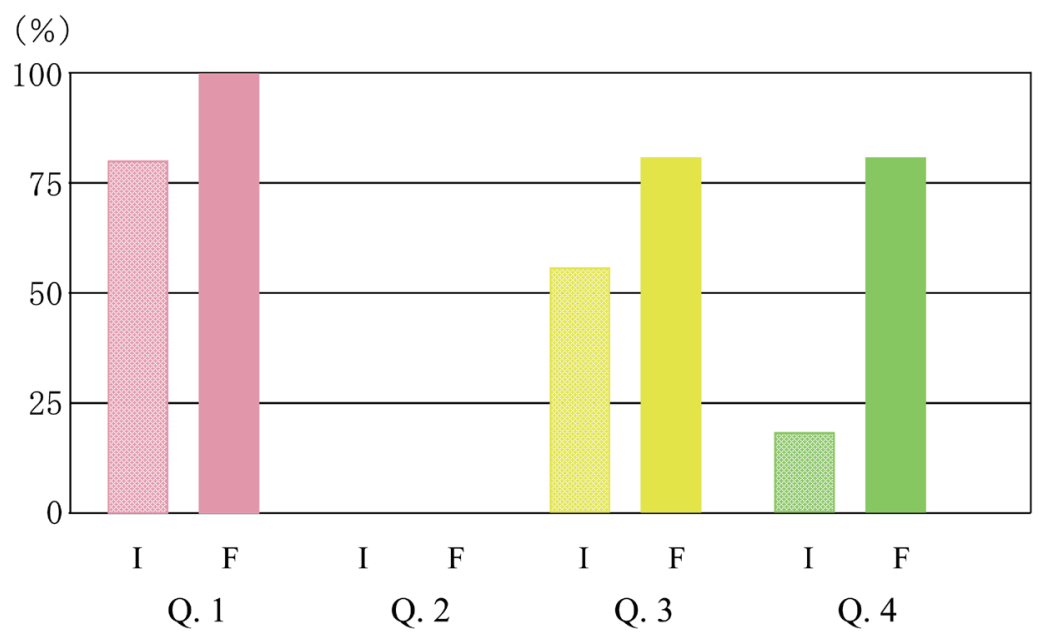

Fig. 10. Healthy children's attitude towards the ND compared to GM. F, final inspection; GM, Glatzel mirror; I, initial inspection; ND, new device with dual cameras for evaluating expiratory nasal flow; Q., question.

at investigating how the ND under development is received by $2-6$ year-old children.

Of all the cleft lip and palate patients, $80.0 \%$ accomplished the assessment successfully at the initial inspection and $95.0 \%$ at the final inspection. Successful assessment accomplishment increased as the assessment progressed. Regarding the children aged 2 years, only $25.0 \%$ completed the assessment successfully at the initial and $75.0 \%$ at the final inspection. All subjects $(100 \%)$ above the age of 3 years completed the assessment successfully, except for the subjects aged 4 years at the initial inspection (Fig. 11).

Of all the healthy children, $71.4 \%$ successfully accomplished the assessment at the initial inspection and $90.5 \%$ at the final inspection. Successful assessment accomplishment also increased as the assessment progressed. At the initial inspection, $44.4 \%$ of the children aged 2 years successfully completed the assessment and $77.8 \%$ completed at the final inspection. In this group, subjects aged 3, 4, and 5 years successfully accomplished all assessments (Fig. 12).

The results of sex-specific attitude towards the ND are shown in Fig. 13. The rates of successful assessment accomplishment for both sexes improved as the trial progressed. Similar tendency was observed in both, cleft lip and palate patients and healthy subjects.

\section{DISCUSSION}

Generally, palatoplasty is performed when the patients are approximately 2 years old, and afterwards speech therapy is continued for several years to prevent VPI. Accurate assessment of velopharyngeal function is 


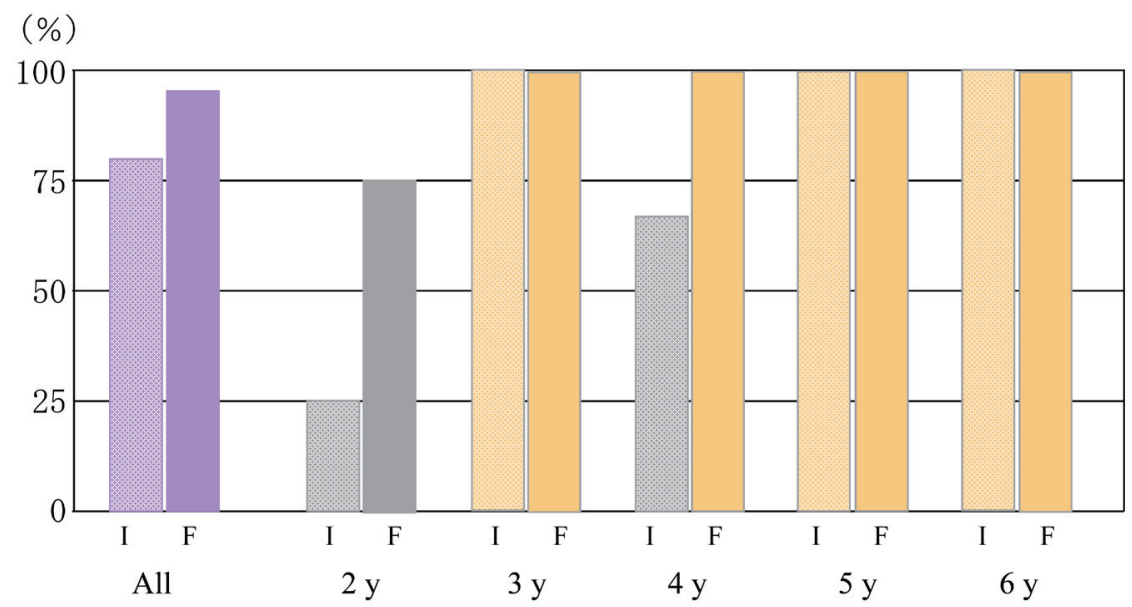

Fig. 11. Age-specific attitude towards the ND in cleft lip and palate patients. After age 3, all subjects indicted $100 \%$ successful assessment, except for those aged 4 years at initial inspection. F, final inspection; I, initial inspection; ND, new device with dual cameras for evaluating expiratory nasal flow; $y$, years old.

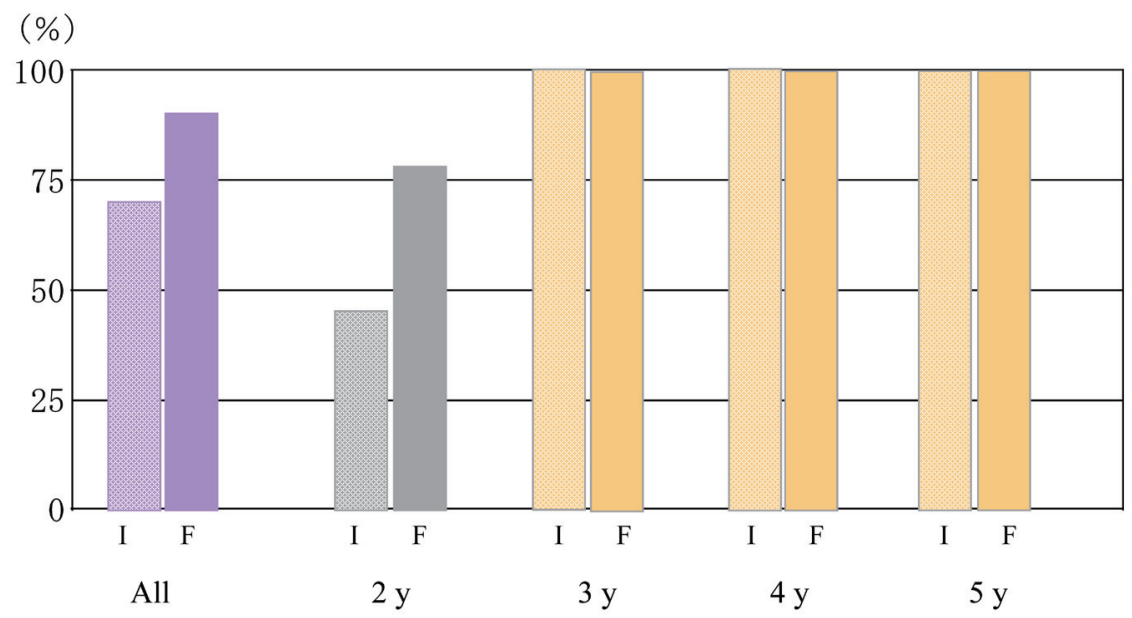

Fig. 12. Age-specific attitude towards the ND in healthy children. Children aged 3, 4, and 5 years successfully accomplished all assessments. F, final inspection; I, initial inspection; ND, new device with dual cameras for evaluating expiratory nasal flow; y, years old.

necessary to determine the correct treatment strategies to ensure optimum speech function. This function can be assessed by various methods and we have to choose the most suitable method according to each case. ${ }^{1-10,14}$ Among these methods, the GM is a non-invasive technique with convenience of use, and can be used in multiple facilities, including clinical and non-clinical environments. It has also been recommended by the Japanese Association of Communication Disorders so that information can be smoothly exchanged between institutions when necessary. ${ }^{7}$

In this study, we focused on mirror testing method based on the GM for the accurate assessment of expiratory nasal flow, and compared it to a digital recording technique using a newly developed device. Additionally, we evaluated the use of this device in post-palatoplasty children from 2 to 6 years of age. Several studies based on the principle of the GM have been previously reported, which included a liquid crystal rhinomanometer that changes color in response to changes in temperature, ${ }^{15}$ and a photo-rhinometer that can evaluate nasal patency under natural respiration. ${ }^{16}$ However, in these studies, all the subjects were adults and large measuring apparatus was involved because of separation of the GM from a camera or video recording device. Such large measuring devices may induce fear and inhibition of interest in children. Moreover, cleft lip and palate patients have to continue speech training after the evaluation of VPI, therefore, the device needs to be received by children. Thus, we investigated the ND, consisting of two digital 

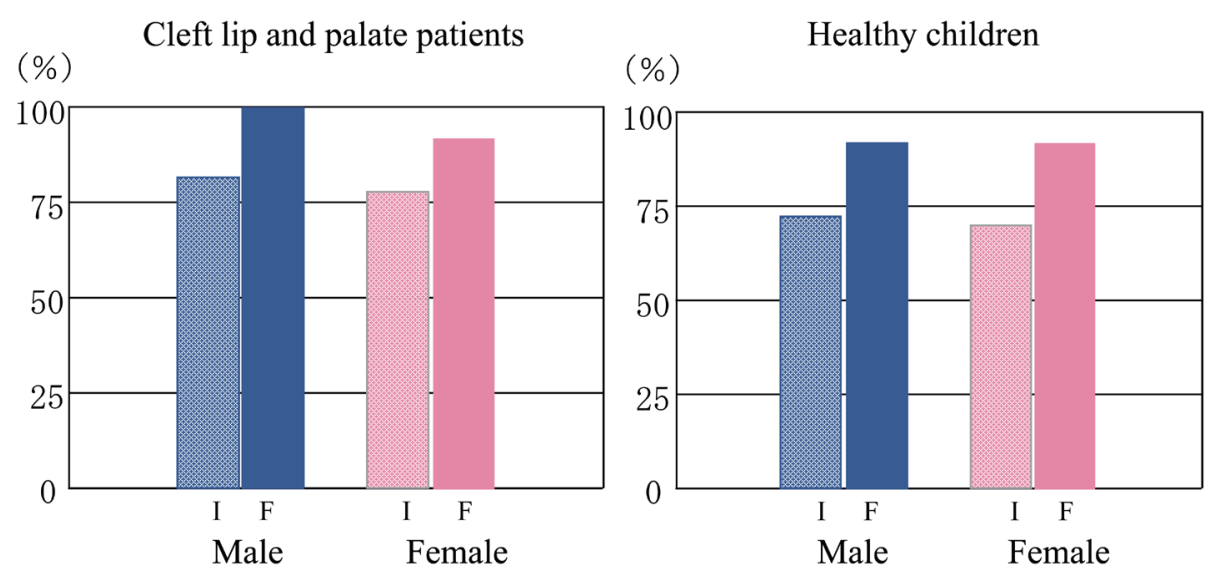

Fig. 13. Sex-specific attitude towards the ND. The rates of successful assessment accomplishment for both sexes improved as the trial progressed. F, final inspection; I, initial inspection; ND, new device with dual cameras for evaluating expiratory nasal flow.

cameras and tulip ornamentation to determine if it had improved expiratory nasal flow assessment capabilities and improved attitudes of patients towards its use. The images produced by the cameras were overlaid on each other to produce an admissible assessable image.

It is difficult to compare the identical pronouncing measurement value of the GM to that of the ND because of different conditions, therefore we compared not only the CA values but also the SVA values of the ND to the GM values. And we put the attention about the case that had the measurement value of the SVA and CA of the ND was greater than the GM in this study. In contrast, if the value of the GM was greater than the SVA and CA of the ND, it was considered inadmissible. This is because it only shows vague measurement values of the GM due to difficult regulation of condensation flow and the short time frame for evaluation. The results of this comparison indicated that the CA of the ND was significantly more accurate than the assessment with GM. This is to say, the ND is significantly superior to the GM in the assessment of expiratory nasal flow of children.

In the questionnaire to measure the feelings of the subjects towards the ND, positive attitudes towards use were categorized as (excellent / good / possible). Over $75.0 \%$ of cleft lip and palate children and healthy children responded positively during the initial inspection. As the research progressed positive responses, such as "I used very well" and "I want to do again" increased, in both cleft lip and palate patients and healthy children group. No subjects from the 2 groups responded "I felt scared" after the final assessment. These results indicated that the ND, consisting of 2 digital cameras and tulip ornamentation, was adopted by the subjects in this research.
Previously, very few researches have studied the correlation between children's age and sex profile and assessment. ${ }^{10}$ In this study, analysis of the age profile suggests that the non-compliance in assessment of subjects aged 2 years was attributed to immaturity, insufficient comprehension, and understanding of the inspection. However, as the inspection progressed the subjects adapted. Furthermore, all subjects aged 3 years could use the ND from the initial inspection. About the GM, a previous study reported that 3.5-yearold children were capable of using it more easily as blowing test with GM. ${ }^{10}$ From these findings, this study clearly demonstrates that the ND is more adoptable for further evaluation in younger children. Analysis of the sex profile in this study showed that the number of the subjects who could use the ND improved in both male and female subjects as the research progressed, and a similar tendency was observed in both groups.

In conclusion, the aim of this study was to assess the expiratory nasal flow accurately by the ND, which consists of two digital cameras and tulip ornamentation, and to evaluate the children's attitude towards the apparatus. This study showed that the ND could detect the expiratory nasal flow significantly more accurately than the existing GM. Moreover, as the number of inspections increased, the ND was better adopted by the children. According to the analysis of the age profile, all children aged 3 years could use the ND from the initial inspection, it demonstrated that the ND is more adoptable for relatively younger children. From these results, this study indicated the superiority of the ND over the GM for the evaluation of expiratory nasal flow in children. 
Acknowledgments: The authors thank all the patients and participants in this study. Additionally, we would like to express our thanks to Yusuke Yoshida of Tottori Institute of Industrial Technology for technical advice, Naomi Shigetaka of Tottori University Hospital Suginoko Nursery School for study support, and Hiroshi Sunada, Assistant Professor of Tottori University for the advice on statistical analysis. We would like to thank Editage (www.editage.com) for English language editing.

The authors declare no conflict of interest.

\section{REFERENCES}

1 Nakamura N. Evaluation and treatment of speech function of patients with cleft palate. Kagoshima Daigaku Shigakubu Kiyo. 2006;26:9-19. Japanese with English abstract.

2 Suzuki A, Goto K, Nakamura N, Honda Y, Ohishi M, Tashiro $\mathrm{H}$, et al. Cephalometric comparison of craniofacial morphology between primary bone grafted and nongrafted complete unilateral cleft lip and palate adults. Cleft Palate Craniofac J. 1996;33:429-35. DOI: 10.1597/1545-1569_1996_033_0429 ccocmb_2.3.co_2, PMID: 8891375

3 Honda $\bar{Y}$, Suzuki A, Nakamura N, Ohishi M. Relationship between primary palatal form and maxillofacial growth in Japanese children with unilateral cleft lip and palate: infancy to adolescence. Cleft Palate Craniofac J. 2002;39:527-34. DOI: 10.1597/1545-1569_2002_039_0527_rbppfa_2.0.co_2, PMID: 12190341

4 Nakamura N, Suzuki A, Takahashi H, Honda Y, Sasaguri M, Ohishi M. A longitudinal study on influence of primary facial deformities on maxillofacial growth in patients with cleft lip and palate. Cleft Palate Craniofac J. 2005;42:633-40. DOI: 10.1597/03-151.1, PMID: 16241175

5 Ogata Y, Nakamura N, Kikuta R, Matsuzaki S, Sasaguri M, Nakama T, et al. A new trial guidance for speech therapy for subjects woth cleft palate based on analysis of the behaviors of velopharyngeal incompetence. Nihon Kōgairetsu Gakkai zasshi. 2007;32:273-82. Japanese with English abstract.

6 Sawada M. [Secondary palatoplasty for velopharyngeal insufficiency]. Nihon Kōgairetsu Gakkai zasshi. 1993;18:1524. Japanese.
7 Miura M, Kato M, Tani M, Ohira A, Kitano I, Kimura T, et al. [Inspection of cleft palate speech]. The Japanese. J Commun Disord. 2009;26:230-5. Japanese.

8 Kato H, Naito K, Horibe S, Horibe H, Nagashima K, Hirai E. Aerodynamic evaluation of velopharyngeal incompetence in children with cleft palate. Fujian Med J. 2016;2:80-2.

9 Soma Y, Hirai E, Horibe S, Horibe T, Naito K. Usefulness of nasometers for evaluation of velopharyngeal competence in children with cleft lip and palate. Shōni Jibi Inkōka. 2018;39:320-6. Japanese with English abstract.

10 Uchiyama T, Motohashi Y, Yamamoto K, Watanabe H, Koeda H, Nakano Y, et al. Methods of assessing velopharyngeal function in cleft palate patients. Shikwa Gakuho. 1999;99:641-56. Japanese with English abstract.

11 Yoshida Yu, Akagami S, Ikuta M, Cyuriki N, Doi R. Pilot study: Speech therapy using a nasal air leakage in the mirror test equipped camera. Nihon Kōgairetsu Gakkai zasshi. 2018;43:158. Japanese.

12 Kondo K, Doi R, Ryoke K. Image synthesis of twin camera using self similarity for nasal breath test. IEICE Technical Report. 2015;3:5760. Japanese with English abstract.

13 Nakahara K, Mishiba K, Doi R, Kondo K. Nasal expiration image detection on mirror surface using alternating optimization. Proceedings of the $7^{\text {th }}$ IIAE International conference on industrial application engineering. 2019;233-8. DOI: DOI: 10.12792/iciae2019.045.

14 Oka S, Kubota Y, Nakagawa S, Takenoshita Y, Ogata Y, Shirasuna K. Relationship between velopharyngeal function and velopharyngeal form. Nihon Kōgairetsu Gakkai zasshi. 2002;27:1-6. Japanese with English abstract.

15 Haruna S, Ozawa M, Uchida Y, Moriyama H. Application of a liquid crystal rhinomanometer to nasal health examination. Jibi Rinsyo. 1997;90:165-71. Japanese with English abstract.

16 Yasuhara A, Nozue M. [Development and evaluation of a photo-rhinometer for clinical use]. Nihon Jibiinkoka Gakkai Kaiho. 1999;102:1287-95. DOI: 10.3950/jibiinkoka.102.1287, PMID: 10655717 